\title{
Microflow fields in the hinge region of the CarboMedics bileaflet mechanical heart valve design
}

Hwa-Liang Leo, MS ${ }^{a}$

Zhaoming $\mathrm{He}, \mathrm{PhD}^{\mathrm{b}}$

Jeffrey T. Ellis, PhD ${ }^{\mathrm{a}}$

Ajit P. Yoganathan, $\mathrm{PhD}^{\mathrm{b}}$

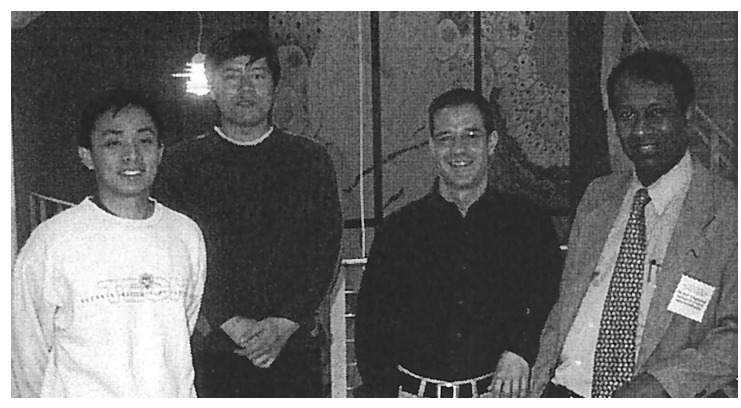

Leo, He, Ellis, Yoganathan (left to right)
Objective: The design of bileaflet mechanical heart valves includes some degree of leakage flow on valve closure for the reverse flow to wash the hinge and pivot region of the valve. It is believed that this reverse flow helps to prevent areas of stasis and inhibit microthrombus formation. However, the magnitude of this retrograde flow may also give rise to unacceptable levels of blood element damage and lead to platelet activation or hemolysis as a result of the increased flow velocities through the hinge region. The purpose of this study was to evaluate the hinge flow dynamics of a 23-mm CarboMedics bileaflet mechanical valve (Sulzer CarboMedics Inc, Austin, Tex) and then to compare the results with those of the St Jude Medical 23-mm Regent (St Jude Medical Inc, Minneapolis, Minn) and Medtronic Parallel (Medtronic, Inc, Minneapolis, Minn) valves studied earlier. This comparison allows new insight into the microflow fields within the hinge region of the CarboMedics bileaflet mechanical valve, which have not been previously assessed during its clinical history.

Methods: Two-dimensional laser Doppler velocimetry was used to measure the velocity and turbulent shear stress fields in the hinge regions. To conduct these measurements, exact dimensional models of the bileaflet hinge regions were cast or machined from transparent plastic materials. The experiment was conducted in a pulsatile flow loop with measurements taken at different levels within the pivot and hinge regions.

. $^{\mathrm{a}}$ the School of Mechanical EngineerBiomedical Engineering, ${ }^{\text {b }}$ Cardiovascular Fluid Mechanics Laboratory, Georgia Institute of Technology, Atlanta, Ga.

This study was funded by a research grant from St Jude Medical Inc, Minneapolis, Minn.

Received for publication Oct 19, 2001; revisions requested Jan 4, 2002; revisions received Feb 26, 2002; accepted for publication March 2, 2002.

Address for reprints: Ajit P. Yoganathan, MD, Associate Chair, School of Biomedical Engineering, Georgia Institute of Technology, 315 Ferst Dr, IBB Building, Room 1121, Atlanta, GA 30332-0535 (E-mail: ajit.yoganathan@bme.gatech.edu).

J Thorac Cardiovasc Surg 2002;124:561-74

Copyright (C) 2002 by The American Association for Thoracic Surgery

0022-5223/2002 \$35.00+0 12/1/125206

doi:10.1067/mtc.2002.125206
Results: In the 23-mm CarboMedics valve hinge, the phase-averaged forward velocity obtained at the flat level and levels of $190 \mu \mathrm{m}$ and $390 \mu \mathrm{m}$ above flat and $1 \mathrm{~mm}$ below flat were $0.54 \mathrm{~m} / \mathrm{s}, 0.77 \mathrm{~m} / \mathrm{s}, 0.3 \mathrm{~m} / \mathrm{s}$, and $1.0 \mathrm{~m} / \mathrm{s}$, respectively. Corresponding values of the peak phase-averaged leakage velocities were $3.17 \mathrm{~m} / \mathrm{s}$, $2.91 \mathrm{~m} / \mathrm{s}, 2.52 \mathrm{~m} / \mathrm{s}$, and $0.5 \mathrm{~m} / \mathrm{s}$, respectively. Corresponding turbulent shear stresses were $5510 \mathrm{dyne} / \mathrm{cm}^{2}, 5640 \mathrm{dyne} / \mathrm{cm}^{2}, 4380 \mathrm{dyne} / \mathrm{cm}^{2}$, and $4810 \mathrm{dyne} / \mathrm{cm}^{2}$, respectively.

Conclusions: The hinge flow dynamics of the CarboMedics bileaflet design lie somewhere in between those of the St Jude Medical and the Medtronic Parallel valve designs. The fluid dynamics of the investigated valve were found to be similar to those of the St Jude Medical valves, although with slightly higher leakage velocities and turbulent shear stresses. This discrepancy may be a result of the sharper corners associated with the hinge design of the CarboMedics valve. It could also be due to the incremental enlargement of the internal orifice area of the St Jude Medical Regent design. 


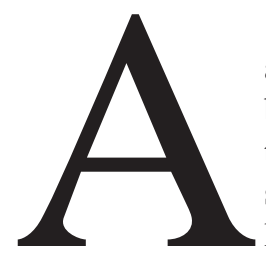

certain degree of regurgitant flow is usually incorporated into the design of a bileaflet mechanical heart valve to ensure the washout of critical areas of the valve, such as hinges and regions between the leaflet edges and housing (Figures 1 and 2). This washout is intended to prevent flow stasis that may be created in the vicinity of the hinge regions and upstream of the valve housing during valve closure in an attempt to minimize, if not eliminate, the formation of thrombosis, which still today poses a significant problem. Recent studies have shown the importance of the bileaflet mechanical heart valve hinge geometry in influencing the characteristics of the leakage flow structures, such as the leakage velocities and turbulent shear stresses (TSSs), ${ }^{1-5}$ within and near the hinge region. The constricted flow area at the hinge region of these mechanical heart valves, coupled with the highpressure difference across the bileaflet valves during closure, is a location in which the fluid can be subjected to high TSSs. ${ }^{6}$ These studies have suggested the hinge mechanism as the most critical part of the bileaflet mechanical heart valve, because it directly influences the valve durability, functionality, and thrombus formation.

\section{Valves of Interest}

\section{Medtronic Parallel}

Although the Medtronic Parallel (MP) mechanical heart valve (Medtronic, Inc, Minneapolis, Minn) was discontinued in clinical trials a few years ago, a hinge flow study was performed on it, and the results were compared with those from other mechanical heart valve studies to illuminate the effects of hinge geometry on the leakage flow characteristics. ${ }^{1-3,6-9}$ Its washout at the hinge regions is considered inferior to that of the St Jude Medical (SJM) Standard, Hemodynamic Plus (HP), and SJM Regent bileaflet mechanical heart valve designs (St Jude Medical Inc, Minneapolis, Minn) and, as shown in this study, to that of the CarboMedics prosthetic heart valve (CPHV) bileaflet design (Sulzer CarboMedics Inc, Austin, Tex). The MP valve leaflets are held in place by four cylindrical projections on the leaflets. The leaflet projections are placed into the four recesses, which are machined into the valve housing near the flat surface on the inflow side of the valve.

The geometry of the MP valve hinge recess is characterized by sudden expansion and contraction zones, which may give rise to flow that is dominated by unsteady flow, vortex structure, and zones of stagnation, and these are the flow phenomena usually associated with elevated level of TSSs. TSSs recorded near the hinge wall in the inflow channel were greater than $6000 \mathrm{dyne} / \mathrm{cm}^{2}$, which is sufficient to cause damage to the blood elements and lead to the activation of thrombosis. ${ }^{3}$ The damaged blood elements entrapped by these vortical fluid structures in the hinge region make the subsequent cyclic washout less effective, thus contributing to the potential buildup of more damaged blood elements. In this study two regions of the hinge were highlighted as suspected locations of thrombus formation: (1) the leaflet projections, where separate zones of high TSS and flow stasis may be created during the systolic phase, and (2) the inflow channel, where high TSS near the hinge wall may cause damage to the platelets or red blood cells.

\section{St Jude Medical}

In contrast to the performance of the MP valve, the SJM bileaflet valve has presented over time a better design in terms of long-term rates of thrombogenicity and valverelated events. The SJM hinge geometry is characterized by a semicircular projection on the leaflet, which mates to a similarly shaped recess in the valve housing. The streamlined geometry of the hinge provides a smoother hinge flow, effectively minimizing the formation of unwanted vortex structures and complex flow seen in the MP valve. ${ }^{3}$ The highest peak leakage velocity and TSS recorded in the SJM $25-\mathrm{mm}$ standard valve were $3.5 \mathrm{~m} / \mathrm{s}$ and $7200 \mathrm{dyne} / \mathrm{cm}^{2}$, respectively, ${ }^{7}$ whereas an improved equivalent model, the SJM 23-mm Regent valve, achieved lower leakage velocity and TSS of $1.5 \mathrm{~m} / \mathrm{s}$ and 2600 dyne $/ \mathrm{cm}^{2}$, respectively. ${ }^{1}$ However, with the SJM valve there is a region of expansion, called the thumbnail, situated downstream of the hinge mechanism. The thumbnail is a region of slight expansion machined into the housing of the SJM valves that we believe might act as a means of increasing the orifice area. Studies $^{1,2}$ have shown that the thumbnail region is characterized by two recirculation zones, which bound the forward flow and prevent the complete washout in that region. In addition to the complex flow in the thumbnail, flow separation and secondary flow were also observed downstream of the thumbnail. These locations where the complex flows were observed may be vulnerable to the formation of thrombus. However, it should be noted that the flow characteristics observed within the thumbnail region might not be due to the expansion zone but could rather be a result of the design of the bileaflet valve. It is possible that the central orifice jet observed upstream of the valve in the SJM valve design may be caused by the observed recirculation flow in the thumbnail. Therefore the flow characteristics observed in the thumbnail may not be unique to the SJM valve design.

\section{CarboMedics Prosthetic Heart Valve}

Another widely investigated heart valve is the CPHV, which shares many similar design features with the SJM bileaflet valve. The CPHV, a second-generation pyrolytic carbon bileaflet valve with rotatable sewing ring, was first introduced into clinical use in 1986. Ever since its inception, and as the third bileaflet valve to reach clinical application after the SJM and Edwards-Duromedics valves, the CPHV 

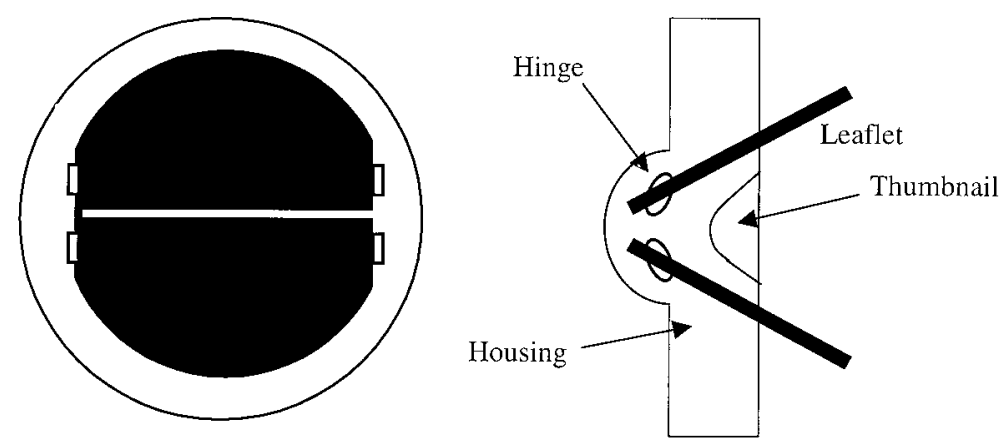

Figure 1. Schematic of generic bileaflet mechanical heart valve showing housing, hinges, and leaflets.
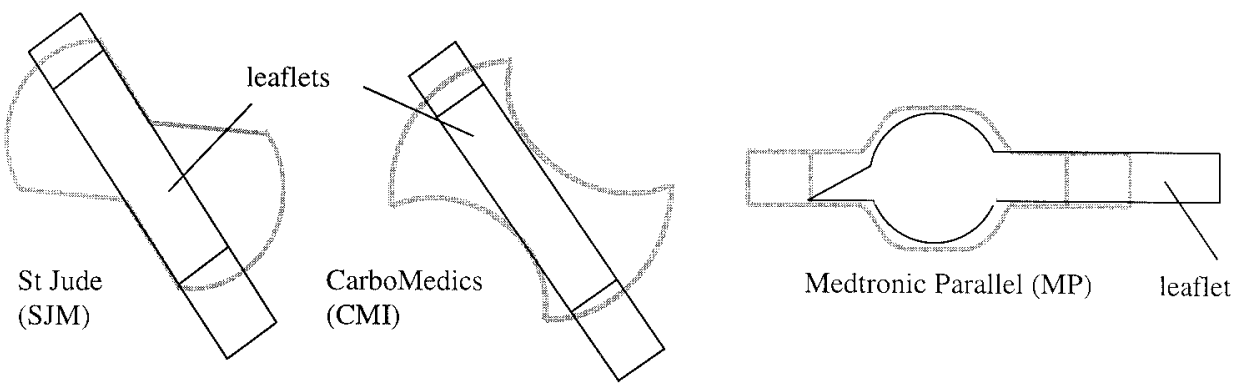

Figure 2. Various hinge geometry designs.

has demonstrated a good clinical history. A study by Dalrymple-Hay and associates ${ }^{10}$ demonstrated that the operative mortality rate of the CPHV compared favorably with those of other mechanical valves, including SJM, Medtronic Hall, and Omniscience. They were also able to show that the CPHV exhibited a low incidence of valve-related complications. In another study, Jamieson and colleagues ${ }^{11}$ showed that CPHVs implanted in mitral, aortic, and multiple positions had satisfactory overall clinical performance. In a study by Aagaard and coworkers ${ }^{12}$ done over a time frame of 12 years, the CPHVs were found to be highly reliable, as evidenced by a total lack of mechanical failures and a low incidence of valve-related complications.

Like the SJM mechanical valves, the CPHV is a lowprofile bileaflet prosthesis with pyrolytic carbon leaflets. There are some differences between these two valves, however; in the SJM bileaflet valves, the leaflets open to $85^{\circ}$ with a travel $\operatorname{arc}$ of $55^{\circ}$ in all sizes, whereas those of the CPHV open to $80^{\circ}$ with a travel arc of $55^{\circ}$. The CPHV has a butterfly hinge geometry similar to that of the SJM valve except that its geometric profile has sharper corners.

Although the difference is statistically insignificant, ${ }^{13}$ higher incidence of valve thrombosis in early and midterm follow-up studies have been reported for the CPHV than for the SJM valve. However, many clinical valve studies do not place much emphasis on the influence of the valve's geometric design, instead focusing mainly on clinical factors, such as the preoperative defective valve seen in native endocarditis, and other nonclinical factors, such as management of anticoagulation. These clinical studies leave many questions involving the influence of valve design unanswered. To fill the gaps left by these clinical studies, this study attempted to provide a detailed fluid dynamic evaluation of the valve in terms of its various design features. It was the aim of this study to provide a fluid dynamic assessment of the CPHV and a comparison of its performance to that of other valves to isolate the factors that may contribute to the performance of the valve. This study provides the first in vitro detailed investigation into the hinge of the CPHV bileaflet design conducted under physiologic conditions. The study forms part of a continued and integrated assessment of the hinge flow characteristics of several bileaflet mechanical heart valves studied previously by Ellis and colleagues. ${ }^{1-3,6,7}$ This investigation was able to improve on the spatial resolution of the laser Doppler velocimetric (LDV) measurements to provide a more detailed assessment of the hinge flow profile.

\section{Methods}

St Jude Medical provided a reverse-engineered casted housing of a clinical quality 23-mm CPHV bileaflet mechanical heart valve. The clear valve housing is a high-quality reproduction of the clinical valve, to ensure an accurate representation of the hinge flow fields to be studied. An analysis of steady leakage study 


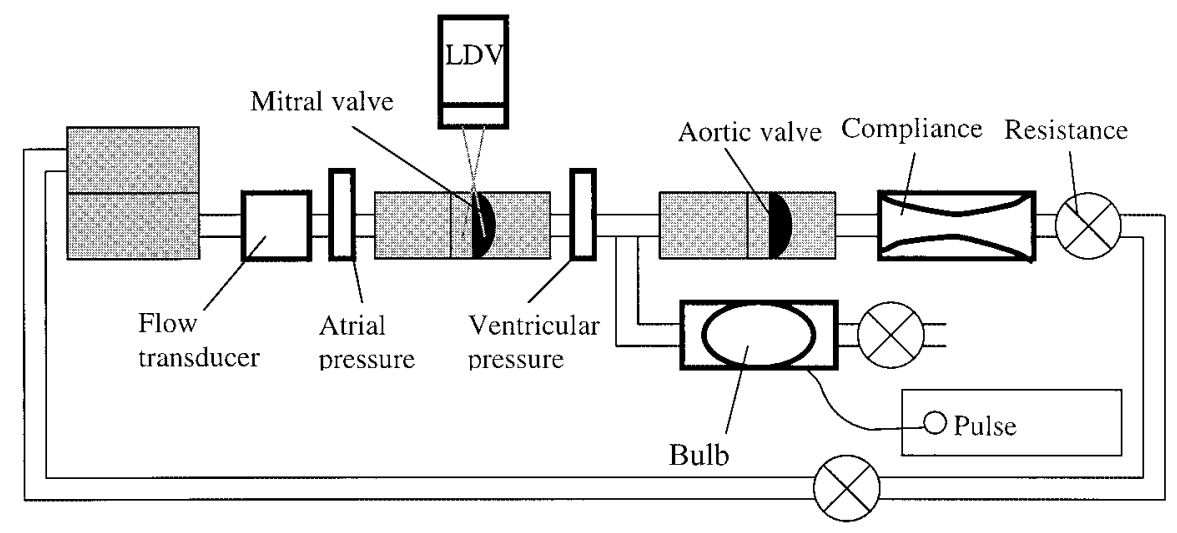

Figure 3. Schematic of pulsatile loop.

showed that the clinical and modeled valves had equivalent leakage flow rates. The casted valve was mounted in the mitral position of the Georgia Tech left heart simulator. The flow loop, shown in Figure 3, was driven by a pulse generator and consisted of tubing, mitral and aortic valves, a flow transducer, atrial and ventricular pressure transducers, a bulb pump, compliance, and resistance.

The flow setup used in this study was typical for a human adult. The specific physiologic conditions for investigating the valve were as follows: heart rate of 70 beats/min (corresponding to an $860 \mathrm{~ms}$ cardiac cycle time), cardiac output of $4.0 \mathrm{~L} / \mathrm{min}$, peak diastolic flow rate of $10.0 \mathrm{~L} / \mathrm{min}$, diastolic duration of $560 \mathrm{~ms}$, and peak left ventricular pressure of $130 \mathrm{~mm} \mathrm{Hg}$. The compliance and resistance sections of the loop allowed additional control of the flow and pressure waveforms. Figure 4, A, shows the mitral flow waveform used in this study (the flow trace was acquired while using the CPHV). It can be seen from the plot that systole began at time interval (or time bin) 41 and ended at time bin 11, whereas diastole began at time bin 12 and ended at time bin 40. Flow rates were measured with an ultrasonic flow probe (model T108/24N; Transonic Systems, Inc, Ithaca, NY), and pressures were measured with a pressure transducer and amplifier (Validyne CD12A1A; Validyne Engineering, Northridge, Calif).

The working fluid was a solution of $79 \%$ saturated aqueous sodium iodide, $20 \%$ glycerin, and $1 \%$ water by volume. This fluid had a kinematic viscosity of $3.5 \mathrm{cSt}$ (centistokes) to match the viscosity of blood at high shear rates. The fluid's refractive index was adjusted to match that of the clear valve housing (1.49), thereby minimizing beam distortion related to changes in refractive index. Silicon carbide particles (TSI Incorporated, St Paul, Minn) with a nominal diameter of $1.5 \mu \mathrm{m}$ were used to seed the flow.

\section{Laser Doppler Velocimetry}

A state-of-the-art, fiber-optic, three-component, coincident LDV system (Aerometrics Inc, Sunnyvale, Calif) was used to obtain two-component velocity and TSS measurements. Two-dimensional LDV measurement was used in the study because of the restriction of the hinge geometry size, which greatly limited the data rate at which data could be collected. In this system, a 4-W argon-ion laser was coupled to a fiber drive unit, allowing color separation of the incoming primary beam. The resulting green (514.5-nm wavelength) and blue (488-nm wavelength) beams were used for the two-component measurements. A Bragg cell was used to add a $40-\mathrm{MHz}$ frequency shift to one beam of each color pair. A two-component fiber-optic transceiver probe with a $100-\mathrm{mm}$ focal length lens was coupled to the fiber drive. The resulting optics train produced an ellipsoid probe volume with minor and major axes of approximately $21 \mu \mathrm{m}$ and $140 \mu \mathrm{m}$, respectively.

Doppler signals were processed with fast Fourier transformbased real-time signal analyzers (Aerometrics). A software package provided by Aerometrics was used to acquire data and to control both the signal analyzers and the photomultiplier hardware. Setting to coincident, all measurements were conducted in backscatter mode, in which a single probe acts as both the transmitter and receiver for the Doppler signals. This mode is used to facilitate the accessibility of the laser beams at the hinge region. An interface was created between a resettable clock and the pulse duplicator system to allow the synchronization of velocity measurements with the mitral flow and ventricular pressure waveform.

\section{Measurement Sites}

Figure $4, B$, shows the top view of the hinge region of the clear housing valve model and the coordinate system that was used. The distances between measurement locations were $0.127 \mathrm{~mm}$ in the $\mathrm{x}$-axis and $0.254 \mathrm{~mm}$ in the $\mathrm{y}$-axis. The resolution chosen provided a detailed and accurate representation of the fluid profile within the hinge region. A total of approximately 21,500 measurements were taken at each location. LDV measurements were performed on the following four planes, as illustrated in Figure 4, $C$ :

- $1 \mathrm{~mm}$ below the primary flat (92 locations)

- Level with primary flat (168 locations)

- $190 \mu \mathrm{m}$ depth into the hinge socket (146 locations)

- $390 \mu \mathrm{m}$ depth into the hinge socket (110 locations)

\section{Mapping of Hinge Geometry}

The hinge geometry was first mapped visually at the primary flat level by moving the laser beams to the edge of the hinge until a bending of the beams was observed. With the hinge boundary coordinates obtained in the first mapping as a reference, a subsequent, finer mapping of the hinge was done with the pulsatile loop 
Bin Number

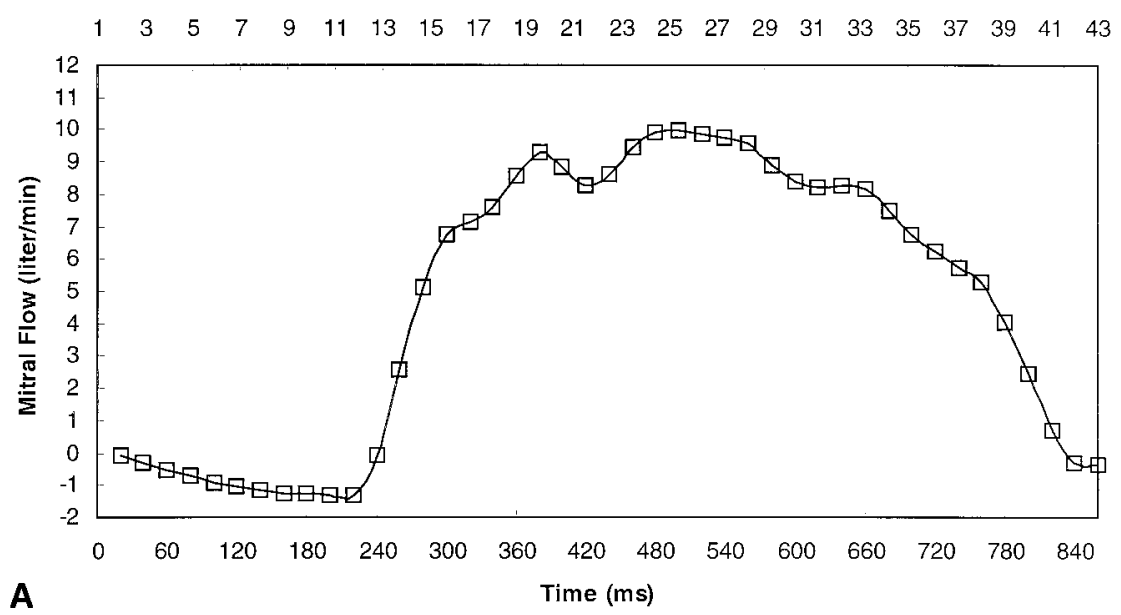

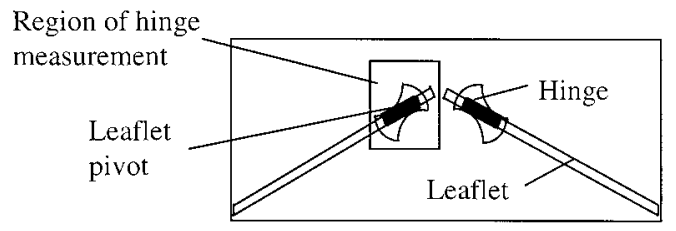

B

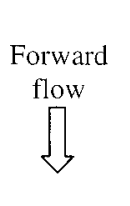

(Depth)

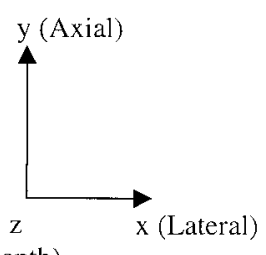

Figure 4. A, Mitral flow waveform as displayed on data-collection computer monitor. B, Schematic of CPHV 23-mm valve and hinge. C, Measurement sites at four different levels within the CPHV hinge.

running. In this mapping, a display of low-frequency signal on an oscilloscope signified the wall boundary. It should be noted that the unit used in Figure 5 is 0.001 inch. Thus, the distance from the lateral to the adjacent corner of the hinge is approximately 0.15 inch, or $3.81 \mathrm{~mm}$. The hinge geometry was remapped each time that measurements were made at a different level, which explains the differences in the geometric coordinates observed at the three levels.

\section{Data Reduction}

Measured velocities were computed off-line, with transit timeweighted averaging to eliminate velocity bias. Velocity bias occurs when a larger or smaller average volume of fluid passes through the probe volume during periods when velocity is faster or slower than the mean. Consequently, a histogram of individual velocity realizations is biased toward both ends of the velocity range.

Velocities were phase-averaged within 20-ms time windows (because each cycle of $860 \mathrm{~ms}$ was divided into 43 time intervals, or bins) for subsequent analysis. A total of approximately 21,500 measurements were made at a single location, an average of approximately 500 measurements within each 20 -ms time window was used for each reduction. This temporal resolution was selected because it was long enough to ensure a valid statistical result in each time window and at the same time short enough for the data set within to be considered quasisteady.

The maximum, or principal, values of the TSSs were determined according to the formulation found in the literature. ${ }^{14-16}$ The TSS values are of particular interest because they provide an indication of the magnitude of mechanical stresses from turbulence) that are experienced by the blood elements, such as red blood cells and platelets, when they travel through the hinge regions of the heart valves.

The averaging procedure is summarized as follows. Within each time window, the axial velocity data was decomposed as follows:

$$
\mathrm{V}_{1}=\overline{\mathrm{V}}_{1}+\nu_{1}^{\prime}
$$




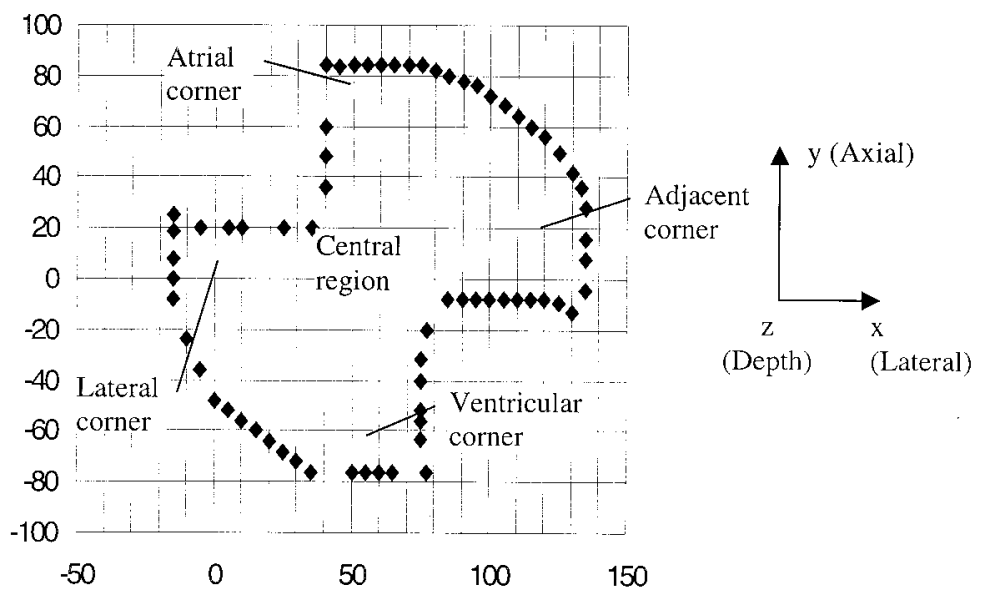

Figure 5. Hinge geometry as mapped by laser at primary flat level.

where $V_{1}$ is an instantaneous axial velocity measurement, $\bar{V}_{1}$ is the mean of all instantaneous $\mathrm{V}_{1}$ measurements in the time window, and $\nu_{1}{ }^{\prime}$ is the fluctuation about this mean value. The same procedure was performed for the instantaneous circumferential $\left(V_{2}\right)$ measurements. Then the total mean velocity magnitude $\left(\bar{V}_{\text {mag }}\right)$ in the time window was calculated as follows:

$$
\overline{\mathrm{V}}_{\text {mag }}=\sqrt{\left(\overline{\mathrm{V}}_{1}^{2}\right)+\left(\overline{\mathrm{V}}_{2}^{2}\right)}
$$

With the mean and instantaneous terms both known, each fluctuating term was calculated along with the TSS $\left(\rho \overline{\nu_{1}^{\prime} \nu_{2}^{\prime}}\right)$ and the two normal TSSs $\overline{\left(\rho \nu_{1}^{\prime} \nu_{1}^{\prime}\right.}$ and $\left.\rho \overline{\rho \nu_{2}{ }^{\prime} \nu_{2}{ }^{\prime}}\right)$. However, the peak TSSs occurring on a fluid element are defined by the principal TSSs at that location. For a two-component system, the two time-averaged principal normal stresses $\left(\overline{\sigma_{\rho 1}}\right.$ and $\left.\overline{\sigma_{\rho 2}}\right)$ and the maximum time-averaged TSS $\left(\bar{\tau}_{\text {max }}\right)$, are given by the following equations:

$\sigma_{\rho 1}=\rho\left[\overline{\frac{\mathrm{V}_{1}{ }^{\prime} \mathrm{V}_{1}{ }^{\prime}}{2}+\overline{\mathrm{V}_{2}{ }^{\prime} \mathrm{V}_{2}^{\prime}}}+\sqrt{\left(\frac{\overline{\mathrm{V}_{1}{ }^{\prime} \mathrm{V}_{1}^{\prime}}-\overline{\mathrm{V}_{2}{ }^{\prime} \mathrm{V}_{2}{ }^{\prime}}}{2}\right)^{2}+\left(\overline{\mathrm{V}_{1}{ }^{\prime} \mathrm{V}_{2}{ }^{\prime}}\right)^{2}}\right]$

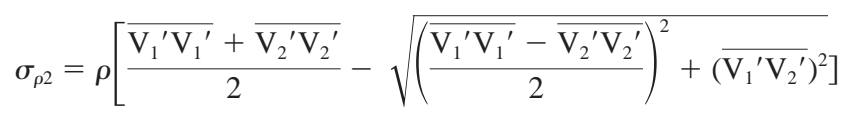

$$
\overline{\tau_{\max }}=\frac{\overline{\sigma_{\rho 1}}-\overline{\sigma_{\rho 2}}}{2}
$$

Throughout the remainder of this article, references to TSS are understood to be the values of $\bar{\tau}_{\max }$ defined in the preceding discussion.

\section{Results}

The results are presented in the form of color pictures in Figures 6 through 18, which illustrate the flow fields at specific instances during the cardiac cycle. The direction of the forward flow is from top to bottom (from left atrium to

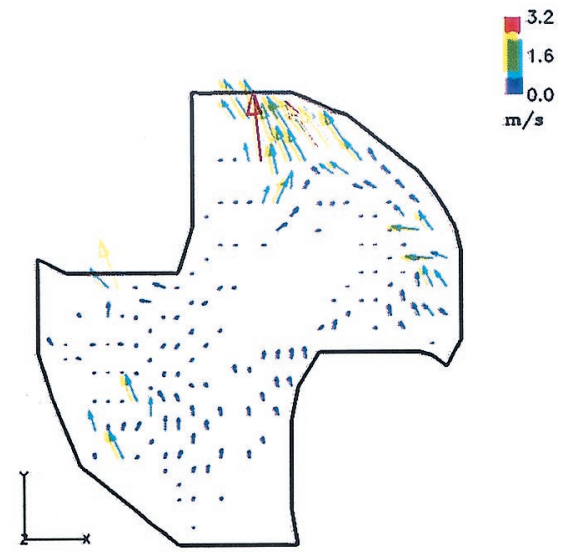

Figure 6. CPHV 23-mm mitral hinge flow field at early systole, flat level. Arrows point in direction of mean velocity vectors and are color-coded by velocity magnitude (in meters per second) as given in key at top right.

left ventricle). Arrows point in the direction of the mean velocity vector and are color coded by velocity magnitude (in meters per second) as noted in the legends. The length of the arrow is proportional to the velocity magnitude.

\section{Flat Level}

Time bin 1 in Figure 6 shows an onset of leakage flow when the leakage jet was observed in the atrial corner of the hinge. Flow was drawn toward the atrial side of the hinge by the backward motion of the leaflet leading to a maximum velocity of $3.17 \mathrm{~m} / \mathrm{s}$ at this region and a corresponding TSS of $5510 \mathrm{dyne} / \mathrm{cm}^{2}$. Another area of high velocity was in the lateral corner of the hinge, where the fluid reached a velocity of approximately $2 \mathrm{~m} / \mathrm{s}$. The velocity in the central region of the hinge was relatively low, in the range of 0.2 to $0.4 \mathrm{~m} / \mathrm{s}$. The TSS magnitudes in the atrial and adjacent 


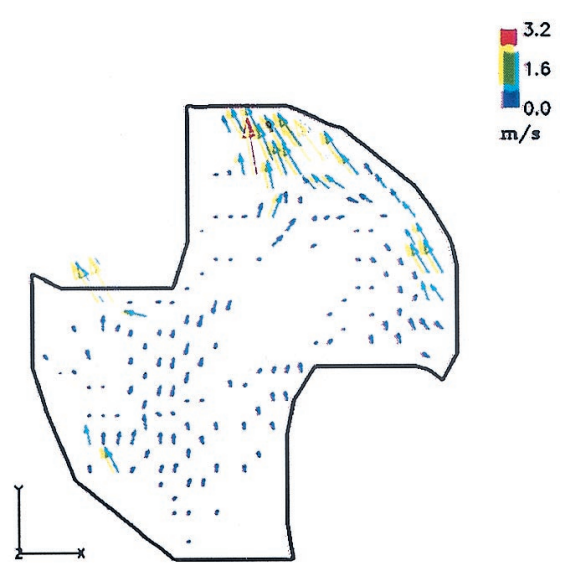

Figure 7. CPHV 23-mm mitral hinge flow field at mid systole, flat level. Arrows point in direction of mean velocity vectors and are color-coded by velocity magnitude (in meters per second) as given in key at top right.

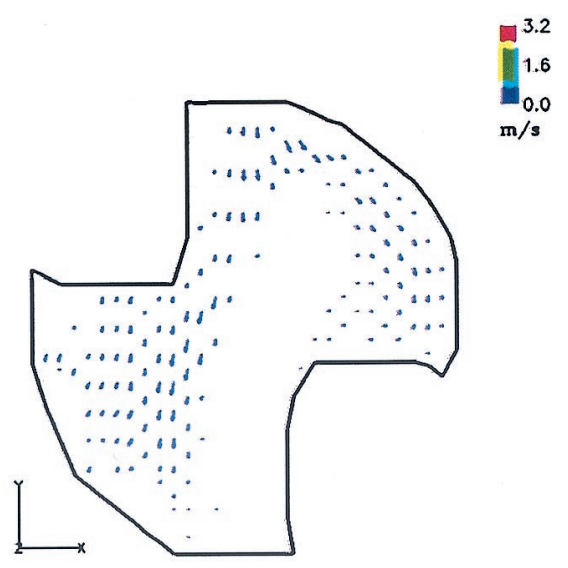

Figure 8. CPHV 23-mm mitral hinge flow field at early diastole, flat level. Arrows point in direction of mean velocity vectors and are color-coded by velocity magnitude (in meters per second) as given in key at top right.

corners of the hinge reached a maximum value of approximately 3000 dyne $/ \mathrm{cm}^{2}$. The TSSs measured near the lateral corner of the hinge were relatively low, at about 300 dyne/ $\mathrm{cm}^{2}$. The flow patterns at time bin 4 in Figure 7 (mid systole) were similar to those at time bin 1 . The leakage jet observed at time bin 4 had decelerated to a velocity of approximately $2.4 \mathrm{~m} / \mathrm{s}$. Here a mixture of forward and leakage flows were observed: flow in the atrial corner of the hinge was predominantly leakage, whereas that in the central region of the hinge was a combination of forward and leakage flows. The peak velocity of the leakage flow through the pivot eventually decreased in magnitude to approximately $1 \mathrm{~m} / \mathrm{s}$. This reduction in velocity extended to the whole hinge region, where the average velocity ob-

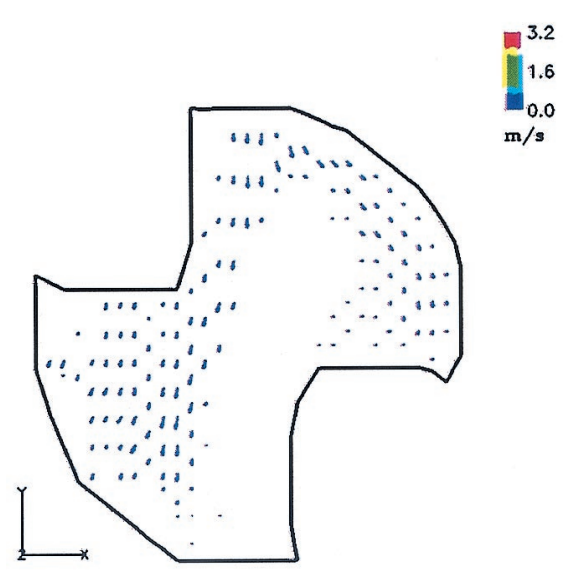

Figure 9. CPHV 23-mm mitral hinge flow field at peak diastole, flat level. Arrows point in direction of mean velocity vectors and are color-coded by velocity magnitude (in meters per second) as given in key at top right.

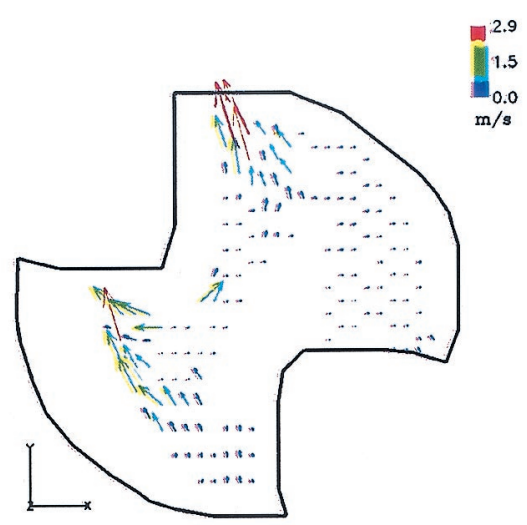

Figure 10. CPHV 23-mm mitral hinge flow field at early systole, 190 $\mu \mathrm{m}$ above flat level. Arrows point in direction of mean velocity vectors and are color-coded by velocity magnitude (in meters per second) as given in key at top right.

served was on the order of $0.01 \mathrm{~m} / \mathrm{s}$. Lack of measurement at both the lateral and ventricular corners of the hinge was due to low data rates in these regions. This could be due to out-of-plane (parallel to z-axis) movement of the fluid particles at these corners.

At time bin 13 (early diastole, Figure 8), flow was predominantly directed forward. A maximum forward velocity of $0.54 \mathrm{~m} / \mathrm{s}$ was observed in the lateral corner of the hinge. The velocity magnitude was approximately 0.2 to 0.3 $\mathrm{m} / \mathrm{s}$, much lower than that seen during leakage flow. Similar flow profiles and magnitudes can be seen at time bin 23 (peak diastole, Figure 9), when velocity within the hinge region was more homogenous (low velocity gradient) at approximately 0.2 to $0.3 \mathrm{~m} / \mathrm{s}$. In both time bins the average value of TSS at most sites had reduced to approximately 


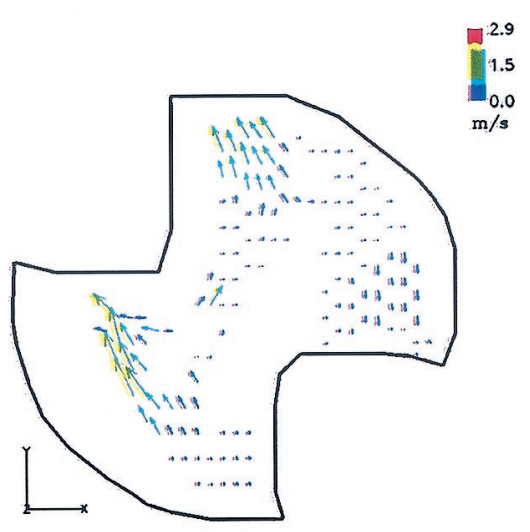

Figure 11. CPHV 23-mm mitral hinge flow field at mid systole, 190 $\mu \mathrm{m}$ above flat level. Arrows point in direction of mean velocity vectors and are color-coded by velocity magnitude (in meters per second) as given in key at top right.

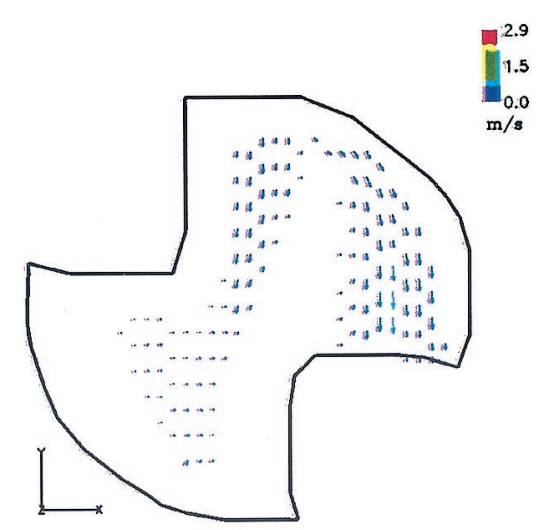

Figure 12. CPHV 23-mm mitral hinge flow field at early diastole, $190 \mu \mathrm{m}$ above flat level. Arrows point in direction of mean velocity vectors and are color-coded by velocity magnitude (in meters per second) as given in key at top right.

$200 \mathrm{dyne} / \mathrm{cm}^{2}$. The lack of measurement in the central region can be attributed to the obstruction cause by the leaflet's protrusion (or pivot) into the sample volume of the velocimeter.

\section{$190 \mu \mathrm{m}$ Above Flat}

At time bin 1 (onset of leakage flow, Figure 10), two distinct jets could be observed at both atrial and lateral parts of the hinge. A peak velocity of $2.91 \mathrm{~m} / \mathrm{s}$ and a corresponding maximum TSS of 5640 dyne $/ \mathrm{cm}^{2}$ were measured at the atrial corner of the hinge, whereas a peak velocity of $2 \mathrm{~m} / \mathrm{s}$ was attained at the lateral corner of the hinge. These regions of leakage flow could lead to the entrainment of the fluid surrounding the hinge. For the remaining parts of the hinge, the velocity field obtained was of relatively low magnitude at approximately 0.05

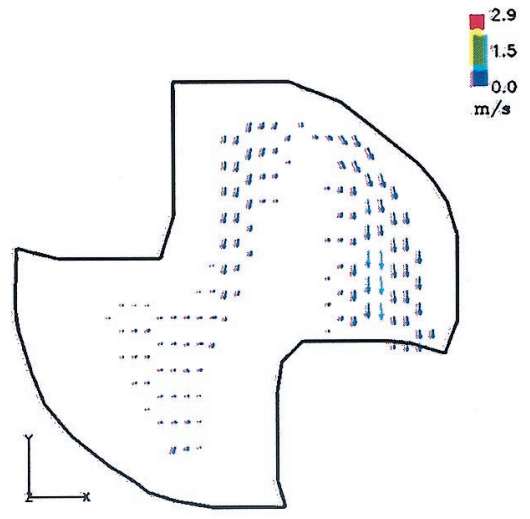

Figure 13. CPHV 23-mm mitral hinge flow field at mid diastole, 190 $\mu \mathrm{m}$ above flat level. Arrows point in direction of mean velocity vectors and are color-coded by velocity magnitude (in meters per second) as given in key at top right.

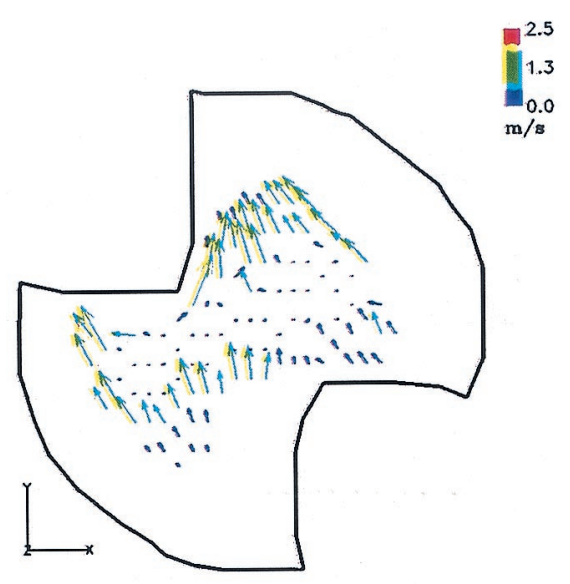

Figure 14. CPHV 23-mm mitral hinge flow field at early systole, 390 $\mu \mathrm{m}$ above flat level. Arrows point in direction of mean velocity vectors and are color-coded by velocity magnitude (in meters per second) as given in key at top right.

$\mathrm{m} / \mathrm{s}$. Isolated regions of high-velocity fluid separated by low-velocity regions may result in extremely high TSSs at their interface in the atrial and lateral corners. The maximum TSS at time bin 1 was approximately 3000 dyne $/ \mathrm{cm}^{2}$ at the atrial corner of the hinge. Another region of high TSS (3000 dyne $/ \mathrm{cm}^{2}$ ) was recorded along the lateral boundary of the hinge. High TSS regions corresponded with those of high velocity gradients. This is because a region with a high velocity gradient, such as that seen in the atrial and lateral corners of the hinge, would produce more shearing of fluid layers, thus leading to higher TSSs. At time bin 5 (mid systole, Figure 11), a reduction in velocity magnitude was observed for the two leakage jets. The peak velocity in the lateral corner of the 


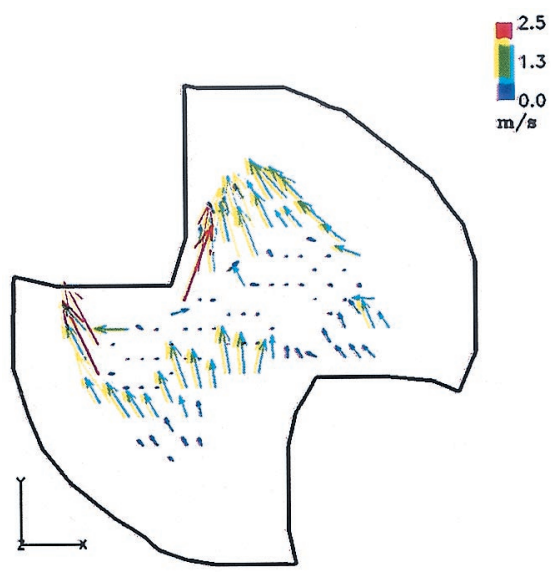

Figure 15. CPHV 23-mm mitral hinge flow field at mid systole, 390 $\mu \mathrm{m}$ above flat level. Arrows point in direction of mean velocity vectors and are color-coded by velocity magnitude (in meters per second) as given in key at top right.

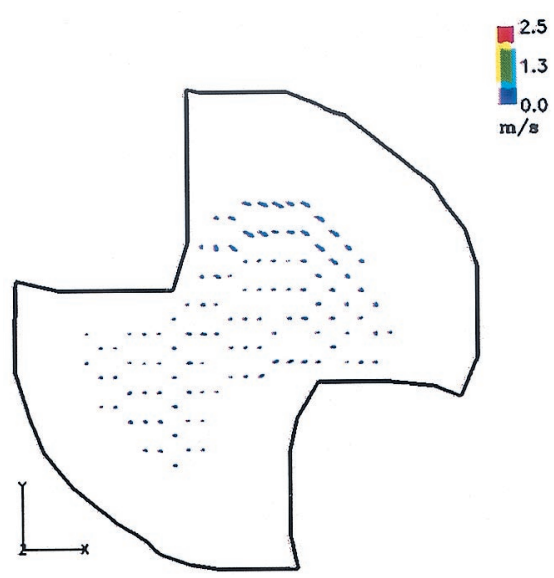

Figure 16. CPHV 23-mm mitral hinge flow field at mid diastole, 390 $\mu \mathrm{m}$ above flat level. Arrows point in direction of mean velocity vectors and are color-coded by velocity magnitude (in meters per second) as given in key at top right.

hinge $(2 \mathrm{~m} / \mathrm{s})$ was higher than that at the atrial corner $(0.9$ $\mathrm{m} / \mathrm{s})$. A region of forward flow $(0.3 \mathrm{~m} / \mathrm{s})$ at the adjacent corner of the hinge began to form at this time bin. Although the leakage jets continued to decelerate at time bin 5 , the forward flow region at the adjacent corner of the hinge continued to increase in size. Three distinct regions of flow were observed at time bin 5, even though the average velocity throughout the hinge was lower.

Time bin 15 (early diastole, Figure 12) was characterized by a prominent forward flow region at the adjacent corner of the hinge. This region began to form around time bin 5 and continued to increase in magnitude toward the end-systolic phase and the beginning of the diastolic phase. This increase extended into time bin 22 (peak diastole, Figure 13), when

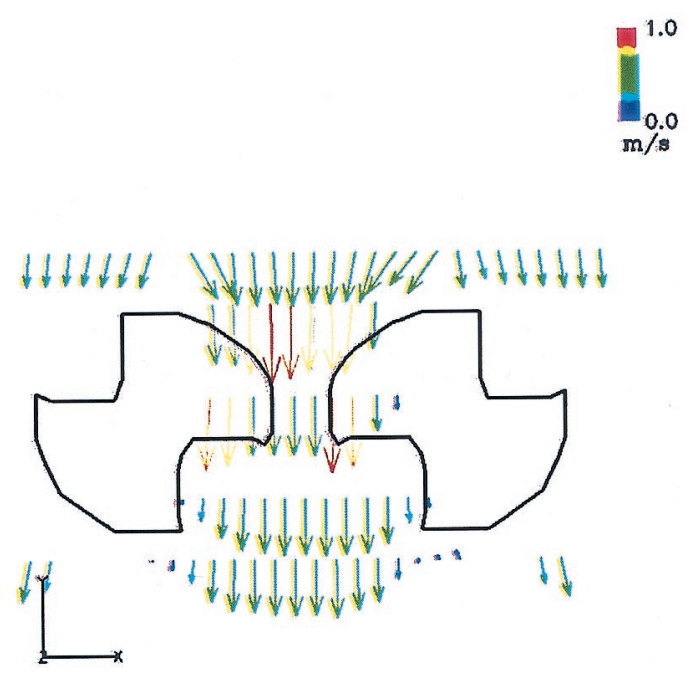

Figure 17. CPHV 23-mm mitral hinge flow field at mid diastole, 1 $\mathrm{mm}$ below flat level. Arrows point in direction of mean velocity vectors and are color-coded by velocity magnitude (in meters per second) as given in key at top right.
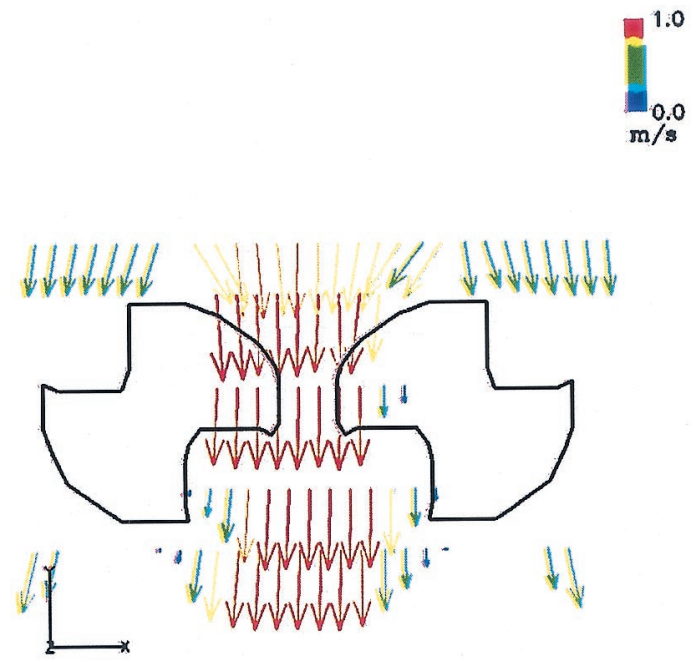

Figure 18. CPHV 23-mm mitral hinge flow field at peak diastole, 1 $\mathrm{mm}$ below flat level. Arrows point in direction of mean velocity vectors and are color-coded by velocity magnitude (in meters per second) as given in key at top right.

it reached a peak velocity of $0.77 \mathrm{~m} / \mathrm{s}$. The central region of the hinge where no measurements were obtained increased in size relative to that seen at the flat level. The leaflet's pivot seems to have extended further up into this part of the hinge socket and blocked off most of the measurement sites. Toward the end of the systolic phase, the values of TSS at most locations decreased to $500 \mathrm{dyne} / \mathrm{cm}^{2}$. This reduction continued well into time bins 15 and 22 (early diastole and mid diastole, Figures 11 and 12, respectively). 


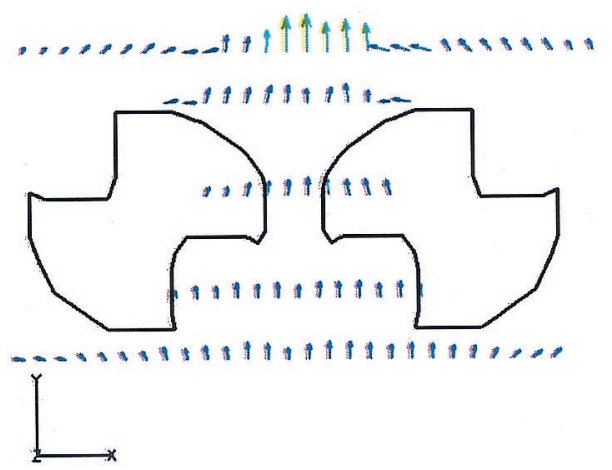

Figure 19. CPHV 23-mm mitral hinge flow field at early systole, 1 $\mathrm{mm}$ below flat level. Arrows point in direction of mean velocity vectors and are color-coded by velocity magnitude (in meters per second) as given in key at top right.

\section{$390 \mu \mathrm{m}$ Above Flat}

At time bin 43 (onset of leakage flow, Figure 14), a streak of leakage jet following the hinge wall boundary could be observed. It was especially pronounced at the atrial corner of the hinge. The leakage jet at this region was connected to other regions of leakage flow along the hinge boundary. Measurement at this level was not obstructed by the leaflet's pivot. Leakage velocity magnitudes of $1 \mathrm{~m} / \mathrm{s}$ were seen at the atrial corner of the hinge. Another region of high leakage flow of $1.5 \mathrm{~m} / \mathrm{s}$ was observed at the ventricular corner of the hinge. The existence of such a high-flow region could be explained by the fact that the fluid flowing into the atrial corner of the hinge was directed over the top of the leaflet pivot. Although measurements were not obstructed by the pivot, the velocities remained relatively low throughout most of the central part of the hinge. At time bin 3 (mid systole, Figure 15), the magnitude of the leakage jet reached a peak velocity of $2.52 \mathrm{~m} / \mathrm{s}$ and a corresponding TSS of 4380 dyne $/ \mathrm{cm}^{2}$ in the lateral corner. The velocity at the central region of the hinge remained relatively low, showing that the flow dynamics at that region were still much affected by the pivot. At time bin 43, a peak TSS of 2000 dyne $/ \mathrm{cm}^{2}$ was observed at both the atrial and ventricular boundaries of the hinge. The TSS increased at time bin 3 , reaching a value of $2500 \mathrm{dyne} / \mathrm{cm}^{2}$. At time bin 3 (mid systole, Figure 15), higher TSSs were observed in the upper boundary of the hinge. At time bin 16 (mid diastole, Figure 16), the reduced leakage flow was replaced by a relatively low peak forward flow of $0.3 \mathrm{~m} / \mathrm{s}$ at the atrial corner of the hinge. The values of TSS at most sites had decreased to approximately $500 \mathrm{dyne} / \mathrm{cm}^{2}$.

\section{$1 \mathrm{~mm}$ Below Flat}

It can be seen from the figures that the direction and magnitude of the flow vectors were the result of the position of the leaflets rather than that of the hinge location. At time bin 14 (mid diastole, Figure 17), a region of forward flow began to form at the center between the two hinge sockets. The maximum velocity observed was approximately $0.8 \mathrm{~m} / \mathrm{s}$ on the atrial side of the hinge region, whereas two lateral jets of approximately $0.5 \mathrm{~m} / \mathrm{s}$ were seen directed over to the sides of the hinge. An increase in magnitude of the central jet could be observed at time bin 23 (Figure 18), when it reached a maximum velocity of $1.0 \mathrm{~m} / \mathrm{s}$. As the flow approached the end of the systolic phase and into the start of the diastolic phase, however, the energy of the central jet dissipated and reached a low velocity of $0.25 \mathrm{~m} / \mathrm{s}$. At time bin 42 (Figure 19), at the beginning of the systolic phase, a leakage flow of approximately $0.2 \mathrm{~m} / \mathrm{s}$ could be seen surrounding the hinge. A central jet with a peak velocity of 0.5 $\mathrm{m} / \mathrm{s}$ was formed through the gap between the two leaflets near the atrial corner of the hinge. The highest TSS of 4810 dyne $/ \mathrm{cm}^{2}$ was obtained during the diastolic phase. Two regions of high stress were observed, one at the central leakage jet and another at the right lateral corner. Despite these regions of high TSS, the TSS of the leakage flow had an average value of about $300 \mathrm{dyne} / \mathrm{cm}^{2}$.

\section{Discussion}

The goal of this study was to evaluate the hinge flow dynamics of the CPHV 23-mm bileaflet mechanical heart valve design. Table 1 summarizes the peak values for the velocities within the hinge region of the 23-mm CPHV and the corresponding TSSs. Table 2 summarizes the peak values for the velocity and the corresponding TSS within the hinge regions of the different bileaflet valve designs.

\section{General Features of CPHV Hinge and Near-Hinge Flow Fields}

For purposes of comparison, the flow field measurements in the CPHV hinge study were made in selected regions at locations similar to those used in the previous SJM valve hinge studies. ${ }^{2}$ It should be noted that static leakage velocities obtained for the clear valve housing approximated 0.66 $\mathrm{L} / \mathrm{min}$, which is similar to values obtained for the equivalent pyrolytic carbon valve. ${ }^{5,17}$ The leakage flow field within the hinge region of the CPHV was characterized by a dynamic washout flow pattern, which effectively minimized the formation of vortex structures and complex flows. During systole, the highest leakage velocity and its corresponding TSSs tended to occur in the regions near the atrial and lateral corners of the hinge geometry. These peak values usually occurred the moment after the impact of the valve leaflets against the valve housing during closure. It is postulated that this sudden closure of the valve leaflets may 
contribute to the effective washout of any deposited blood elements or pooled coagulative factors in the in vivo situation.

The forward flow velocities observed in the CPHV were comparable to those observed in the SJM Regent valve. During the forward flow phase, the peak velocities through the hinge were in the range of 0.3 to $0.77 \mathrm{~m} / \mathrm{s}$, with the highest forward flow velocity observed at the 390- $\mu \mathrm{m}$ level. Forward flow velocities were typically low, mostly on the order of $0.3 \mathrm{~m} / \mathrm{s}$ in areas close to the boundary of the hinge. This was because most of the forward flow was through the opened leaflets, with less volume being directed into the hinge. In the $190-\mu \mathrm{m}$ level, three distinct regions of flow were observed during mid systole, even though the average velocity throughout the hinge was lower. The forward flow at the adjacent corner of the hinge was observed only at the $190-\mu \mathrm{m}$ level within the hinge. This may be because the flow of the fluid particles at this level, unlike those at 390 $\mu \mathrm{m}$ and primary flat, were being blocked and directed over the side of the leaflet's pivot. This channeled flow may have caused the elevated velocity magnitudes observed at this phase of the cycle.

The CPHV valve lacks the thumbnail region, so the measurement at $1 \mathrm{~mm}$ below the flat level showed only the hinge region. The flow velocity at $1 \mathrm{~mm}$ below the flat level was relatively low in both the forward and backward directions. The flow at this level was outside the hinge, where the highest velocity magnitude was observed close to the central gap between the two leaflets. The flow arrows in Figures 16 through 18 demonstrate that the position of the leaflets had a more dominant effect on the flow at this level than it did in the levels within the hinge. The energy of the leakage jet within the hinge may have already dissipated before it could have any visible effect on the flow $1 \mathrm{~mm}$ below flat. It is possible that a measurement level closer to the primary flat might show the influence of the hinge flow. Two regions of high stress were observed at this level, one at the central leakage jet and another at the right lateral corner. The former may have been due to the decreasing gap area between the closing leaflets, whereas the latter may have been a result of the constricting flow area between the housing wall and the moving leaflet. However, this was not observed at the left lateral side of the flow, which means that this might be unique to the particular valve studied. This slight flow asymmetry observed at the $1 \mathrm{~mm}$ below flat could have been due to asynchronous leaflet motion.

\section{Influences of Geometry on Hinge Flow Fields in Different Designs}

Studies ${ }^{3,8,9}$ conducted on the MP valve design had suggested that the abrupt changes in the hinge geometry lead to the formation of stagnation sites and regions of elevated levels of turbulence. These studies recorded high leakage velocity
TABLE 1. Peak phase-averaged velocities and TSS values measured within the hinge region of the $23-\mathrm{mm}$ CPHV

\begin{tabular}{|c|c|c|c|}
\hline Elevation & $\begin{array}{c}\text { Peak phase } \\
\text { averaged } \\
\text { forward flow } \\
\text { velocity }(\mathrm{m} / \mathrm{s} \text { ) }\end{array}$ & $\begin{array}{l}\text { Peak phase } \\
\text { averaged } \\
\text { leakage } \\
\text { velocity }(\mathrm{m} / \mathrm{s})\end{array}$ & 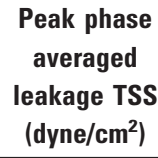 \\
\hline Flat & 0.54 & 3.17 & 5510 \\
\hline $190 \mu \mathrm{m}$ above & 0.77 & 2.91 & 5640 \\
\hline $390 \mu \mathrm{m}$ above & 0.3 & 2.52 & 4380 \\
\hline $1 \mathrm{~mm}$ below & 1.0 & 0.5 & 4810 \\
\hline
\end{tabular}

TABLE 2. Peak leakage velocities and TSS values measured within the hinge regions of the different bileaflet designs

\begin{tabular}{|c|c|c|}
\hline & $\begin{array}{l}\text { Peak phase-averaged } \\
\text { leakage velocity }(\mathrm{m} / \mathrm{s})\end{array}$ & $\begin{array}{l}\text { Peak phase-averaged } \\
\text { leakage TSS }\left(\text { dyne } / \mathrm{cm}^{2}\right)\end{array}$ \\
\hline 27-mm MP & 4.0 & 8000 \\
\hline $\begin{array}{l}\text { 23-mm SJM SHP } \\
\text { (Regent) }\end{array}$ & 1.5 & 2600 \\
\hline 23-mm CPHV & 3.17 & 5640 \\
\hline
\end{tabular}

and TSS of $4 \mathrm{~m} / \mathrm{s}$ and 8000 dyne $/ \mathrm{cm}^{2}$, respectively. The SJM Regent valve, on the other hand, demonstrated lower leakage velocity and TSS $\left(1.5 \mathrm{~m} / \mathrm{s}\right.$ and 2600 dyne $\left./ \mathrm{cm}^{2}\right)$. The SJM hinge mechanism has a streamlined and curved profile that allows the fluid traveling through it to follow the contour of the inside surface, thereby effectively reducing the creation of a vortex within the hinge. The CPHV design has a hinge geometry that is similar to that of the SJM valve, except that it has sharper corners and less streamlined edges. It also does not have a true thumbnail region. This study recorded peak velocities during the leakage flow phase inside the CPHV hinge on the order of magnitude of 2.52 to $3.17 \mathrm{~m} / \mathrm{s}$, with the maximum velocity occurring at the flat level. The leakage velocity measured in this experiment was relatively higher than that observed for the SJM Regent 23-mm valve. The Regent model is a recently (1998) improved version of both the SJM standard and SJM HP models, which were introduced for clinical use in the United States in 1977 and 1993, respectively. The SJM Regent model incorporates design features of the previous SJM models, with the major change being the modification of the internal orifice diameter, which leads to larger geometric and effective orifice areas than seen with the SJM standard model with an equivalent tissue annulus diameter. For example, the internal orifice diameter of the SJM 23-mm Regent valve is approximately two sizes larger than that of the SJM 23-mm standard valve. A previous study by Ellis and Yoganathan ${ }^{2}$ showed that the hinge flow dynamics of SJM Regent valve is equivalent to, if not superior to, the dynamics of the SJM HP valve. These findings may have 
been influenced by the increase in orifice diameter in the SJM Regent valve.

The major design differences between the CPHV and the SJM (Regent and standard) valves include the following: (1) differences in the valve hinge geometry, (2) incorporation of pivot guards, (3) enlargement of the internal orifice diameter in the Regent design, (4) variations in gap width tolerances between the different manufacturers, and, probably to a lesser extent, (5) the incorporation of the thumbnail region in the Regent design. The importance of the influence of hinge geometry has been shown by several studies. A recent LDV study by Steegers and coworkers ${ }^{5}$ compared the leakage fluid dynamic characteristics of a standard SJM bileaflet valve and a CPHV bileaflet valve with similar tissue anulus sizes in the mitral position. They reported a higher leakage jet velocity of $1.7 \mathrm{~m} / \mathrm{s}$ and a maximum TSS of 800 dyne $/ \mathrm{cm}^{2}$ for the CPHV design, with corresponding values of $1.0 \mathrm{~m} / \mathrm{s}$ and 450 dyne $/ \mathrm{cm}^{2}$ for the SJM standard design. This is consistent with the measurements obtained in this study. Higher leakage velocities and TSS values were obtained in the hinge region of a $23-\mathrm{mm}$ CPHV valve $(3.17 \mathrm{~m} / \mathrm{s}$ and 5640 dyne $/ \mathrm{cm}^{2}$ ) than those found in a previous study in our laboratory with a $23-\mathrm{mm}$ SJM Regent valve $(1.5 \mathrm{~m} / \mathrm{s}$ and 2600 dyne $/ \mathrm{cm}^{2}$, Ellis and coworkers ${ }^{1-3}$; Table 2). A similar study by Meyer and coworkers ${ }^{18}$ reported velocities and stresses of equivalent orders of magnitude in the two valve designs. They observed a maximum leakage jet velocity of $2.2 \mathrm{~m} / \mathrm{s}$ and a maximum TSS of 3600 dyne $/ \mathrm{cm}^{2}$ for the CPHV and recorded corresponding values of $0.7 \mathrm{~m} / \mathrm{s}$ and 1800 dyne $/ \mathrm{cm}^{2}$ for the standard SJM valve. A closer inspection of the hinge geometry of the two valves revealed more than a subtle difference in the hinge designs. A crosssectional view of the hinge showed that instead of a smooth, curved recess machined into the valve housing, as seen in the SJM valve designs, the CPHV hinge geometry has an angulated recess. In addition, in the CPHV design the leaflet projections within the recess were observed not to sweep all the way to the edge of the hinge geometry. Therefore visible regions of "unmated" spaces were left at each corner of the hinge geometry during both the fully closed and fully open positions of the leaflets. The angulated recess may offer more gap space in between the leaflet projection and the valve housing, thereby allowing more fluid to flow through the hinge during the systolic phase and thus giving rise to higher leakage jet velocities and corresponding TSSs. The presence of these unmated spaces at the hinge corners may further aggravate the leakage problem, leading to elevated values of leakage jet velocities. Unlike the smooth recess of the SJM valve, the angulated recess of the CPHV may also act to disrupt the hinge flow, which may lead to increased TSSs.

The increase in internal orifice diameter between the Regent and CPHV designs may also contribute to some of the observed differences between these valves. It should be noted that the difference in the internal orifice diameter between the 23-mm SJM Regent and CPHV designs is approximately $3 \mathrm{~mm}$. Because the valves have the same tissue annulus but the CPHV has a smaller internal orifice diameter, the closure velocity of the CPHV leaflets should be higher than that of the SJM Regent valve leaflets, which has a larger internal orifice diameter. If this is the case, in the CPHV the volume of fluid flowing upstream during the closing of the leaflets may be insufficient to reduce the pressure difference across the valve, leading to a higher pressure difference and consequently a correspondingly higher leakage velocity during valve closure. On the other hand, in the SJM valve the leaflet closure velocity may be lower, enabling enough backflow during the initial phase of systole, thus effectively reducing the pressure difference and the subsequent leakage velocity across the valve during valve closure. It should be noted that the difference in valve closure velocity can have effects on the leakage velocity during the closing of the valve leaflets and not when they are closed.

Although there are variations in the leakage jet flow rates in each valve design as a result of the variations in the gap width tolerance in the hinge region, the values obtained with the CPHV design were consistently higher than those obtained with the SJM design. The characteristics of the leakage flow in a valve may thus be attributed to a combination of factors that comprise the unique geometry of the hinge, the valve internal orifice diameter, gap tolerances in the hinge region, and unique characteristics, such as the SJM thumbnail expansion downstream of the hinge region.

The magnitude of TSS $\left(\mathrm{dyne} / \mathrm{cm}^{2}\right)$ and the exposure times of the blood elements to the TSS are related to the mean velocity of the flow across the hinge region of the valve. Results from this study as well as others by Ellis and coworkers ${ }^{1-3,6,7}$ have shown that the increases in TSS were accompanied by increases in the mean flow velocity. Stein and Sabbah ${ }^{19}$ have reported that thrombus formation is a result of mechanical turbulence stresses. Essentially, the thrombosis can be derived from the activation of the platelets and the destruction of the red blood cells or hemolysis. Values of the TSSs reported to cause hemolysis range from as low as $400 \mathrm{dyne} / \mathrm{cm}^{2}$ to $5600 \mathrm{dyne} / \mathrm{cm}^{2}$, with exposure times ranging from $10^{-4}$ to $10^{2}$ seconds. ${ }^{20-22}$ The reported values of turbulent stresses leading to the activation of platelets range from $100 \mathrm{dyne} / \mathrm{cm}^{2}$ to $1000 \mathrm{dyne} / \mathrm{cm}^{2}$, with exposure times ranging from $10^{-2}$ to $10^{2}$ seconds. ${ }^{23,24} \mathrm{Re}$ cently, $\mathrm{Lu}$ and colleagues ${ }^{25}$ have set the hemolytic threshold to $800 \mathrm{dyne} / \mathrm{cm}^{2}$, with an exposure time of $1 \mathrm{~ms}$. The varied reported turbulent values can only make sense if these results are interpreted according to the scale of the turbulent eddies created, because it is speculated that turbulent eddies (Kolmogorov scale eddies) smaller or similar in size to the 
blood cells could lead to hemolysis.,26 The Kolmogorov length scale is defined as

$$
\eta=\left(\frac{v^{3} l}{u^{3}}\right)^{1 / 4}
$$

where $v$ is the kinematic fluid viscosity, $l$ is the length scale of the largest eddies, and $u$ is the root mean square velocity as follows:

$$
\mu=\left(\frac{\mathrm{PNS}_{\mathrm{max}}}{\rho}\right)^{1 / 2}
$$

where $P N S_{\max }$ is the maximum principal turbulent normal stress. The maximum principle normal stress obtained at the $190 \mu \mathrm{m}$ above primary flat level was approximately 11,888 dyne $/ \mathrm{cm}^{2}$. With u taken to be $3.5 \times 10^{-6} \mathrm{~m}^{2} / \mathrm{s}$ and the gap width between the ear of the leaflet and the acrylic valve housing taken as $250 \mu \mathrm{m}$, the calculated Kolmogorov length scale, $\eta$, is approximately $10 \mu \mathrm{m}$. The size of the turbulent length scale is on the same order of the characteristic dimensions of platelets and red blood cells, which have sizes of $3 \mu \mathrm{m}$ and $8 \mu \mathrm{m}$, respectively. The results show that the high turbulent stresses that arise within the hinge regions may lead to sublethal or lethal damage to blood elements. Although the results in this study imply that SJM hinge flow is better than the CPHV in an in vitro setting, it is necessary to remember that these findings do not necessarily translate directly to in vivo performance.

\section{Conclusions}

On the basis of the peak leakage velocities and the peak TSSs, this study demonstrates that the hinge flow dynamics of the CPHV lie somewhere in between those of the SJM Regent and the MP bileaflet valve designs. With improved spatial resolution, this study was able to provide a detailed assessment of the flow profile within the hinge region of the CPHV 23-mm valve design. The fluid dynamics of the investigated valve were found to be similar to those of SJM valves, although with higher leakage velocities and TSSs. These differences are mainly attributed to the differences in the hinge geometries of the two valve designs. The findings of this study should be evaluated together with other important performance criteria, such as structural durability and long-term clinical history and outcomes.

\section{Experimental Limitations}

The LDV measurements were conducted only at selected locations and thus do not completely define the flow fields within the hinge regions of the valve. Also, the measurements were acquired in a 2-dimensional plane, and any out-of-plane velocities (change in the orientation of the jet) are not represented. It is possible that these confined flow fields may indeed be 3-dimensional because of the complex geometries formed by the mating of the leaflet and hinge.
Other experimental techniques, ${ }^{4,9}$ such as high-speed flow visualization or the use of scaled up models, may be useful in assessing the flow fields more completely. The primary experimental limitations that prevented more detailed investigations were the optical access of the models and the width of the gap between each leaflet and housing. The low data rate sometimes encountered in the measurement contributed to the cyclic variation error for the TSS. Furthermore, the aforementioned sites are close to where the leaflet mates against the hinge stop when opening or closing. In such a small, confined area it is possible that vibrations or pressure wave reflections caused by the impact of the leaflets against the hinge stop caused the probe volume to shift slightly into an adjacent region. Although no study was conducted to investigate the effect of vibration on the velocity measurements, preliminary tests were performed at two levels, namely the 390- $\mu \mathrm{m}$ and $190-\mu \mathrm{m}$ levels within the hinge socket, and the velocity data were shown to be similar to those obtained during the actual experiment. Another limitation of this study is that of the use of rigid, mounted models. However, the simplified setup used helps isolate various factors that might contribute to the influence of the hinge flow dynamics. Further, because we ensured that the waveforms obtained were compatible with physiologic conditions, we are confident that the in vitro setup could to a high degree of approximation mimic the flow dynamics of the in vivo conditions. Although only one set of test valves was used, the steady flow leakage data obtained from the valve was representative of this particular type of valve design, and it should provide reliable flow information with regard to its fluid dynamic performance. Steady flow leakage tests were performed on three sets of valves, and the results were shown to be similar. In addition, although the clear housing valve was a reproduction of a clinical quality CPHV 23-mm bileaflet valve, the dimensional accuracy of the reproduction should be considered in evaluating the limitations of this study. As stated earlier, the static leakage flow rate through the reproduced clear housing valve was similar to that through the clinical quality carbon valve, thereby providing confidence in the reproduction technique. Furthermore, our previous experience with this experimental technique leads us to believe that the transparent valve models do accurately reproduce the critical dimensions of the hinge regions of the clinical quality valves.

\section{References}

1. Ellis JT, Travis BR, Yoganathan AP. An in vitro study of the hinge and near-field forward flow dynamics of the St Jude Medical Regent bileaflet mechanical heart valve. Ann Biomed Eng. 2000;28:524-32.

2. Ellis JT, Yoganathan AP. A comparison of the hinge and near-hinge flow fields of the St Jude Medical Hemodynamic Plus and Regent bileaflet mechanical heart valves. J Thorac Cardiovasc Surg. 2000; 119:83-93.

3. Ellis JT, Healy TM, Fontaine AA, Saxena R, Yoganathan AP. Velocity measurements and flow patterns within the hinge region of a 
Medtronic Parallel bileaflet mechanical valve with clear housing. J Heart Valve Dis. 1996;5:591-9.

4. Gao ZB, Hosein N, Dai FF, Hwang NH. Pressure and flow fields in the hinge region of bileaflet mechanical heart valves. J Heart Valve Dis. 1999;8:197-205.

5. Steegers A, Paul R, Reul H, Rau G. Leakage flow at mechanical heart valve prostheses: improved washout or increased blood damage? J Heart Valve Dis. 1999;8:312-23.

6. Ellis JT, Healy TM, Fontaine AA, Weston MW, Jarret CA, Saxena R, et al. An in vitro investigation of the retrograde flow fields of two bileaflet mechanical heart valves. J Heart Valve Dis. 1996;5:600-6.

7. Ellis JT. An in vitro investigation of the leakage and hinge flow fields through bileaflet mechanical heart valves and their relevance to thrombogenesis [dissertation]. Atlanta: Georgia Inst. of Tech.; 1999.

8. Gross JM., Shu MC, Dai FF, Ellis JT, Yoganathan AP. A microstructural flow analysis within a bileaflet mechanical heart valve hinge. J Heart Valve Dis. 1996;5:581-90.

9. Healy TM, Fontaine AA, Ellis JT, Walton S, Yoganathan AP. Visualization of the hinge flow in a 5:1 scaled model of the Medtronic Parallel bileaflet heart valve prosthesis. Exp Fluids. 1998;25:512-8.

10. Dalrymple-Hay MJ, Pearce RK, Dawkins S, Alexiou C, Haw MP, Livesey SA, et al. Mid-term results with 1,503 CarboMedics mechanical valve implants. J Heart Valve Dis. 2000;9:389-95.

11. Jamieson WR, Fradet GJ, Miyagishima RT, Henderson C, Brownlee RT, Zhang J, et al. CarboMedics mechanical prosthesis: performance at eight years. J Heart Valve Dis. 2000;9:678-87.

12. Aagaard J, Tingleff J, Hansen CN, Noergaard MA, Rasmussen CE. Twelve years' clinical experience with the CarboMedics prosthetic heart valve. J Heart Valve Dis. 2001;10:177-84.

13. Akins CW. Results with mechanical cardiac valvular prostheses. Ann Thorac Surg. 1995;60:1836-44.

14. Baldwin JT, Deutsch S, Geselowitz DB, Tarbell JM. Estimation of Reynolds stresses within the Penn State left ventricular assist device. ASAIO Trans. 1990;36:M274-8.
15. Healy TM, Ellis JT, Fontaine AA, Jarrett CA, Yoganathan AP. An automated method for analysis and visualization of laser Doppler velocimetry data. Ann Biomed Eng. 1997;25:335-43.

16. Woo Y, Yoganathan A. Pulsatile flow velocity and shear stress measurements on St. Jude bileaflet valve prosthesis. Scand J Cardiovasc Surg. 1986;20:15-28.

17. Reul H, van Son JA, Steinseifer U, Schmitz B, Schmidt A, Schmitz C, et al. In vitro comparison of bileaflet aortic heart valve prostheses: St. Jude Medical, CarboMedics, modified Edwards-Duromedics, and Sorin-Bicarbon valves. J Thorac Cardiovasc Surg. 1993;106: 412-20.

18. Meyer RS, Deutsch S, Bachmann CB, Tarbell JM. Laser Doppler velocimetry and flow visualization studies in the regurgitant leakage region of three mechanical mitral valves. Artif Organs. 2000;25:292-9.

19. Stein PD, Sabbah HN. Measured turbulence and its effect on thrombus formation. Circ Res. 1974:35:608-14.

20. Rooney JA. Hemolysis near an ultrasonically pulsating gas bubble. Science. 1970;169:869-71.

21. Sallam AM, Hwang NH. Human red blood cell hemolysis in a turbulent shear flow: contribution of Reynolds shear stresses. Biorheology. 1984;21:783-97.

22. Sutera SP, Mehrjardi MH. Deformation and fragmentation of human red blood cells in turbulent shear flow. Biophys J. 1975;15:1-10.

23. Hung TC, Hochmuth RM, Joist JH, Sutera SP. Shear-induced aggregation and lysis of platelets. Trans Am Soc Artif Intern Organs. 1976;22:285-91.

24. Ramstack JM, Zuckerman L, Mockros LF. Shear-induced activation of platelets. J Biomech. 1979;12:113-25.

25. Lu PC, Lai HC, Liu JS. A reevaluation and discussion on the threshold limit for hemolysis in a turbulent shear flow. J Biomech. 2001;34: 1361-4.

26. Liu JS, Lu PC, Chu SH. Turbulence characteristics downstream of bileaflet aortic valve prostheses. J Biomech Eng. 2000;122:118-24. 